\title{
Deposition of the NG2 Proteoglycan at Nodes of Ranvier in the Peripheral Nervous System
}

\author{
Sandra Martin, Angela K. Levine, Zhi Jiang Chen, Yvonne Ughrin, and Joel M. Levine \\ Department of Neurobiology and Behavior, State University of New York at Stony Brook, Stony Brook, New York 11794
}

The node of Ranvier is a complex macromolecular assembly of ion channels and other proteins that is specialized for the rapid propagation of the action potential. A full understanding of the processes responsible for the assembly and maintenance of the node requires first the identification and characterization of the proteins found there. Here we show that NG2, a structurally unique chondroitin sulfate proteoglycan, is a molecular component of the node of Ranvier in the peripheral nervous system. In adult sciatic nerve, NG2 is (1) associated with thin, elongated fibroblast-like cells, (2) on some but not all basal laminae, and (3) at nodes of Ranvier. At the nodes, NG2 is restricted to the nodal gap and is absent from the paranodal or juxtaparanodal region. In dissociated cell cultures of adult sciatic nerve, perineurial fibroblasts but not Schwann cells express NG2 on their surfaces. Approximately $45 \%$ of the total NG2 in peripheral

Many axons of the peripheral nervous system use a saltatory mode of action potential conduction in which current flow is restricted to small gaps in the myelin sheath known as nodes of Ranvier. This adaptation allows for faster conduction of the action potential without a corresponding increase in axon diameter. The node of Ranvier is a complex macromolecular assembly of the voltage-dependent ion channels necessary for the generation of the action potential and of other proteins whose main functions appear to be organizing and maintaining the specialized and distinct membranous subdomains of the nodal region (for review, see Vabnick and Shrager, 1998; Arroyo and Scherer, 2000; Peles and Salzer, 2000; Rasband and Schrager, 2000). For example, the type 6 isoform of the voltage-dependent sodium channels is found at extremely high density at nodes of Ranvier in the rat peripheral nervous system (Caldwell et al., 2000). Interactions between the cytoplasmic domain of sodium channel subunits and ankyrin $\mathrm{G}$ may stabilize these high-density clusters in adult nerve (Kordeli et al., 1995; Malhotra et al., 2000). Similarly, voltage-dependent potassium channels of the Shaker family are excluded from the nodal gap but are present in the juxtaparanodal region of the axon (Wang et al., 1993; Mi et al., 1995). The septate junctions between myelinating glial cells and the axolemma may function to restrict the distribution of channels (Rosenbluth, 1976). The axonal proteins caspr and contactin are likely compo-

Received Feb. 12, 2001; revised July 2, 2001; accepted Aug. 7, 2001.

This work was supported by National Institutes of Health Grant NS21198 (J.M.L.). S.M. was supported in part by a University RAIRE research fellowship. We thank Dr. M. N. Rasband for his assistance with the preparation of teased nerve fibers and Dr. J. Trimmer and Dr. M. Grumet for gifts of antibodies.

Correspondence should be addressed to Dr. Joel Levine, Department of Neurobiology and Behavior, State University of New York at Stony Brook, Stony Brook, NY 11794. E-mail: Joel.Levine@sunysb.edu.

Copyright (C) 2001 Society for Neuroscience $\quad 0270-6474 / 01 / 218119-10 \$ 15.00 / 0$ nerves is in a soluble, rather than particulate, subcellular compartment. NG2 is also present in membrane fractions that also contain high levels of voltage-dependent sodium channels, caspr, and neuron-glia related cell adhesion molecule. These medium-density membranes likely correspond to the nodal and paranodal region of the axon-Schwann cell unit. These results suggest a model in which perineurial fibroblasts secrete or shed NG2, which subsequently associates with nodes of Ranvier. The growth-inhibitory and anti-adhesive properties of NG2 may limit the lateral extension of myelinating Schwann cells as nodes mature. NG2 may also participate in the barrier functions of the perineurial linings of the nerve.

Key words: node of Ranvier; perineurium; nerve-blood barrier; chondroitin sulfate proteoglycan; NG2; extracellular matrix

nents of these junctions, but their glial binding partners remain unknown (Einheber et al., 1997; Menegoz et al., 1997; Rios et al., 2000). Other proteins enriched at nodes of Ranvier include cell adhesion and extracellular matrix molecules (Rieger et al., 1986; Martini et al., 1990; Davis et al., 1996). Given the functional importance of the node of Ranvier and the disastrous consequences of demyelinating diseases that disrupt nodal structure, it is important to identify molecular components of the node and to understand their functions there.

Proteoglycans are major constituents of peripheral nerves and have been implicated in the regulation of axon growth and regeneration (Fitch and Silver, 1997). Because proteoglycans can interact with both extracellular matrix molecules and cell surface molecules, the deposition of these multifunctional molecules at nodes may help organize these complex structures.

To further our understanding of the functions of proteoglycans in the peripheral nervous system, we have examined the distribution of the NG2 chondroitin sulfate proteoglycan (CSPG) in adult rat sciatic nerve. NG2 is a well characterized integral membrane proteoglycan found principally on the surfaces of oligodendrocyte precursor cells (OPCs) in the CNS (Levine and Nishiyama, 1996). OPCs send processes to nodes of Ranvier in the CNS (Butt et al., 1999), although the significance of these cellular processes is not known. Here we show that NG2 is present in adult rat sciatic nerve. Immunofluorescence, cell culture experiments, and biochemical analysis suggest a model in which NG2 is synthesized and secreted by perineurial fibroblasts and subsequently associates with nodes of Ranvier and several basal laminae. By virtue of its anti-adhesive and growth-inhibiting properties (Dou and Levine, 1994; Fidler et al., 1999), NG2 at nodes of Ranvier may function to limit the lateral extension of the 
Schwann cell during the late stages of myelination and of node maturation.

\section{MATERIALS AND METHODS}

Immunofluorescence. Adult rats were anesthetized with ketaminexylazine and decapitated, and the sciatic nerve was rapidly removed. After a brief rinse in ice-cold PBS, whole nerves were fixed in freshly prepared $4 \%$ paraformaldehyde in $0.1 \mathrm{M}$ phosphate buffer, $\mathrm{pH} 7.4$, at $4^{\circ} \mathrm{C}$ for not more than $1 \mathrm{hr}$ and then cryoprotected by immersion in $30 \%$ sucrose and $0.1 \mathrm{~m}$ phosphate buffer. Ten micrometer sections were cut at a longitudinal or frontal plane using a cryostat.

For the detection of specific antigens, the following antibodies were used: NG2, rabbit anti-NG2 and mouse monoclonal antibody D31.10 (Levine and Card, 1987); myelin basic protein, monoclonal antibody 382 (Chemicon, Temecula, CA); p75 low-affinity neurotrophin receptor, antibody 1554 (Chemicon); thy1.1 antigen, monoclonal antibody 1406 (Chemicon); ankyrin G, monoclonal antibody 4G3F8 (Zymed, San Francisco, CA); S100 $\beta$ protein, monoclonal antibody SH-B1 (Sigma, St. Louis, MO); neuron-glia related cell adhesion molecule ( $\mathrm{NrCaM})$, rabbit antisera 837 (M. Grumet, Rutgers State University of New Jersey, Piscataway, NJ); all known mammalian sodium channel isoforms, monoclonal antibody K58/35.1 (Rasband et al., 1999; a gift from J. Trimmer, State University of New York, Stony Brook, NY); and caspr, rabbit anti-caspr (J. Trimmer). Antibodies against laminin B2 chain (monoclonal antibody D18) and s-laminin (antibody C4) were obtained from the Developmental Studies Hybridoma Bank (Iowa City, IA). Immunofluorescence staining methods were similar to those described previously (Levine and Card, 1987; Levine et al., 1993). Nuclei were visualized after incubating the sections in Hoechst $33258(0.5 \mu \mathrm{g} / \mathrm{ml}$; Sigma $)$. In single- and double-labeled fluorescence studies, Cy3-conjugated goat anti-mouse (Jackson ImmunoResearch, West Grove, PA) and FITCconjugated goat anti-rabbit (SBTA; Fisher Scientific, Pittsburgh, PA) antibodies were used. In the absence of the primary antibodies, no staining was observed with these fluorochrome-conjugated reagents. Sections and cultures were examined either with a Zeiss (Thornwood, NY) Axiovert microscope equipped with phase contrast and fluorescence optics or with a Zeiss Axioplan microscope equipped with fluorescence and Nomarski optics. Images were taken using either film or a digital camera. In some of the figures, the images were pseudocolored and digitally merged using Metamorph image-processing software (Universal Imaging Corp., West Chester, PA). Figure plates were prepared using Adobe Photoshop (Abode Systems, Inc., Mountain View, CA).

Teased sciatic nerve fibers were prepared as described previously (Rasband et al., 1998) and immunofluorescently stained as described above.

Cell culture. Continuous cultures of highly purified Schwann cells were established according to the method of Brockes et al. (1979) and maintained in DMEM (Fisher Scientific) containing 10\% fetal calf serum, 2.5 $\mu \mathrm{M}$ forskolin (Sigma), and $20 \mathrm{ng} / \mathrm{ml}$ recombinant human heregulin- $\beta 1$ (amino acids 176-246; R \& D Systems, Minneapolis, MN). To prepare dissociated cultures of adult rat sciatic nerve, animals were killed with $\mathrm{CO}_{2}$, and the sciatic nerves removed, cleaned of fat and connective tissue, minced, and then incubated for $45 \mathrm{~min}$ at $37^{\circ} \mathrm{C}$ in solutions containing $0.25 \%$ trypsin (Worthington Biochemicals, Freehold, NJ) and $0.2 \%$ collagenase (Sigma). The tissue was washed in serum-containing media and triturated by passage through a series of flame-narrowed Pasteur pipettes. The cells were plated onto poly-L-lysine-coated coverslips in DMEM containing 10\% fetal calf serum. Cultures of newborn rat dorsal root ganglia were established as described previously (Dou and Levine, 1994) except that the medium did not contain nerve growth factor. Immunofluorescence staining of living cells was performed as described previously (Levine et al., 1993). All procedures using animals were approved by the university Institutional Animal Care and Use Committee.

Biochemical methods. SDS-gel electrophoresis and Western blotting were performed as described previously (Levine et al., 1998) using ECL reagents from Amersham Pharmacia Biotech (Arlington Heights, IL). For densitometric measurements, the x-ray films were scanned using a flat-bed scanner (Microtek, Torrance, CA) and quantitated using Metamorph software (Universal Imaging Corp.). To prepare total soluble and particulate fractions of adult sciatic nerve, frozen nerves (Pel-Freeze Biologicals, Rogers, AR) were homogenized in $0.01 \mathrm{M}$ Tris, pH 8.0, $1 \mathrm{~mm}$ EDTA, 2 mM PMSF, $0.1 \mathrm{~mm}$ 1,10-phenanthroline, and $1 \mu \mathrm{g} / \mathrm{ml}$ leupeptin using a Polytron (Brinkmann Instruments, Westbury, NY) and centrifuged at $120,000 \times \mathrm{g}$ at $4^{\circ} \mathrm{C}$ in an Optima TLX ultracentrifuge (Beckman
Instruments, Palo Alto, CA). The pellet was rehomogenized in $1 \%$ SDS, $10 \mathrm{~mm}$ Tris, pH 8.0, and $1 \mathrm{~mm}$ EDTA and boiled for 3-5 min. Any remaining insoluble material was removed by centrifugation. Soluble and particulate extracts of adult rat brainstem white matter were prepared in an identical manner. In additional experiments, sciatic nerve and brainstem white matter was homogenized in solutions of $1 \% \mathrm{NP}-40,0.15 \mathrm{M}$ $\mathrm{NaCl}, 10 \mathrm{~mm}$ Tris, $\mathrm{pH} 8.0$, and protease inhibitors. After $15 \mathrm{~min}$ on ice, the homogenate was centrifuged at $14,000 \times g$ for $10 \mathrm{~min}$, and the supernatant was removed and kept. Extracts were digested with proteasefree chondroitinase ABC (Seikagaku America Inc.) as described previously (Dou and Levine, 1994). Protein was determined using a dyebinding assay (Bio-Rad, Hercules, CA). All biochemical reagents were from Sigma unless noted otherwise.

Subcellular fractionation. One hundred frozen rat sciatic nerves were used per fractionation. The nerves were homogenized in $40 \mathrm{ml}$ of $12 \%$ sucrose in $10 \mathrm{~mm}$ phosphate, pH 7.35, containing $1 \mathrm{~mm}$ EDTA, $2 \mathrm{~mm}$ PMSF, $0.1 \mathrm{~mm}$ 1,10-phenanthroline, and $1 \mu \mathrm{g} / \mathrm{ml}$ leupeptin using a Polytron. Homogenization was complete when no connective tissue was visible. The homogenate was layered over a discontinuous gradient of 20 and $45 \%$ sucrose and centrifuged for $16 \mathrm{hr}$ at $4^{\circ} \mathrm{C}$ and at 26,000 rpm using a Beckman SW27 rotor. The 20/45\% sucrose interface was collected, diluted in $10 \mathrm{~mm}$ phosphate buffer, and centrifuged again at 35,000 rpm $(100,000 \times g)$ for 40 min using a Beckman 42 rotor. The 12/20\% interface was also collected and concentrated as described above. We refer to this material herein as PNS myelin (see Fig. 7). The resulting pellet was resuspended in $12 \%$ buffered sucrose and homogenized using a handheld Dounce homogenizer, eight passes each of A and B pestles. The homogenate was layered over a second discontinuous gradient of 22, 27, and $35 \%$ sucrose. After overnight centrifugation at $26,000 \mathrm{rpm}$ in a Beckman SW27 rotor, all fractions including the pellet were retained. All fractions were concentrated by centrifugation at $100,000 \times g$ and resuspended in $1 \%$ SDS, $10 \mathrm{~mm}$ Tris, $\mathrm{pH} 8.0$, and $1 \mathrm{~mm}$ EDTA. One-fourth of the SDS extracts was denatured at $37^{\circ} \mathrm{C}$, whereas the remainder was denatured by boiling. The light, medium, and heavy membrane fractions correspond to the $12 / 22 \%$ interface, the $22 / 27 \%$ interface, and the $27 / 35 \%$ interface, respectively.

\section{RESULTS}

We used immunofluorescence microscopy with specific antibodies to examine the disposition of NG2 in longitudinal sections of adult rat sciatic nerve. As shown in Figure $1 A$, NG2-like immunoreactivity was associated with several different structures within the nerve. First, immunoreactivity was found at the lateral margins of the nerve, most likely associated with the basal lamina of the epineurial and perineurial sheaths (Fig. 1A, small-headed arrow). Second, thin linear elements within the nerve bound the anti-NG2 antibodies. Some of these elements were intensely stained, whereas others appeared to bind the antibodies less robustly (Fig. $1 A$, wide-headed arrows). Third, NG2 immunoreactivity was found on small puncta and short, dash-like structures that often were oriented perpendicular to the longitudinal axis of the nerve (Fig. $1 A$, narrow arrows). Fourth, NG2 was found on the blood vessel-like structures lying within the central regions of the nerve (Fig. 1A, wide arrowhead). At higher magnification (Fig. $1 B, D)$, individual NG2-positive profiles appeared as long, thin cells, a characteristic of perineurial fibroblasts in peripheral nerve (Peltonen et al., 1987). Each of these thin, elongated NG2positive profiles contained a Hoechst 33258-positive nucleus, demonstrating that they are cells rather than aggregates of NG2containing extracellular matrix (Fig. 1D,E). These elongated cellular profiles were stained also with an anti-vimentin antibody, as were most of the NG2-negative Schwann cells (data not shown).

To further characterize the NG2-positive cellular profiles, we compared the distribution of NG2 with that of marker antigens specific for Schwann cells using double-label immunofluorescence of the same tissue sections. Figure 2 shows that there was very little overlap in the distribution of NG2 and of myelin-associated 

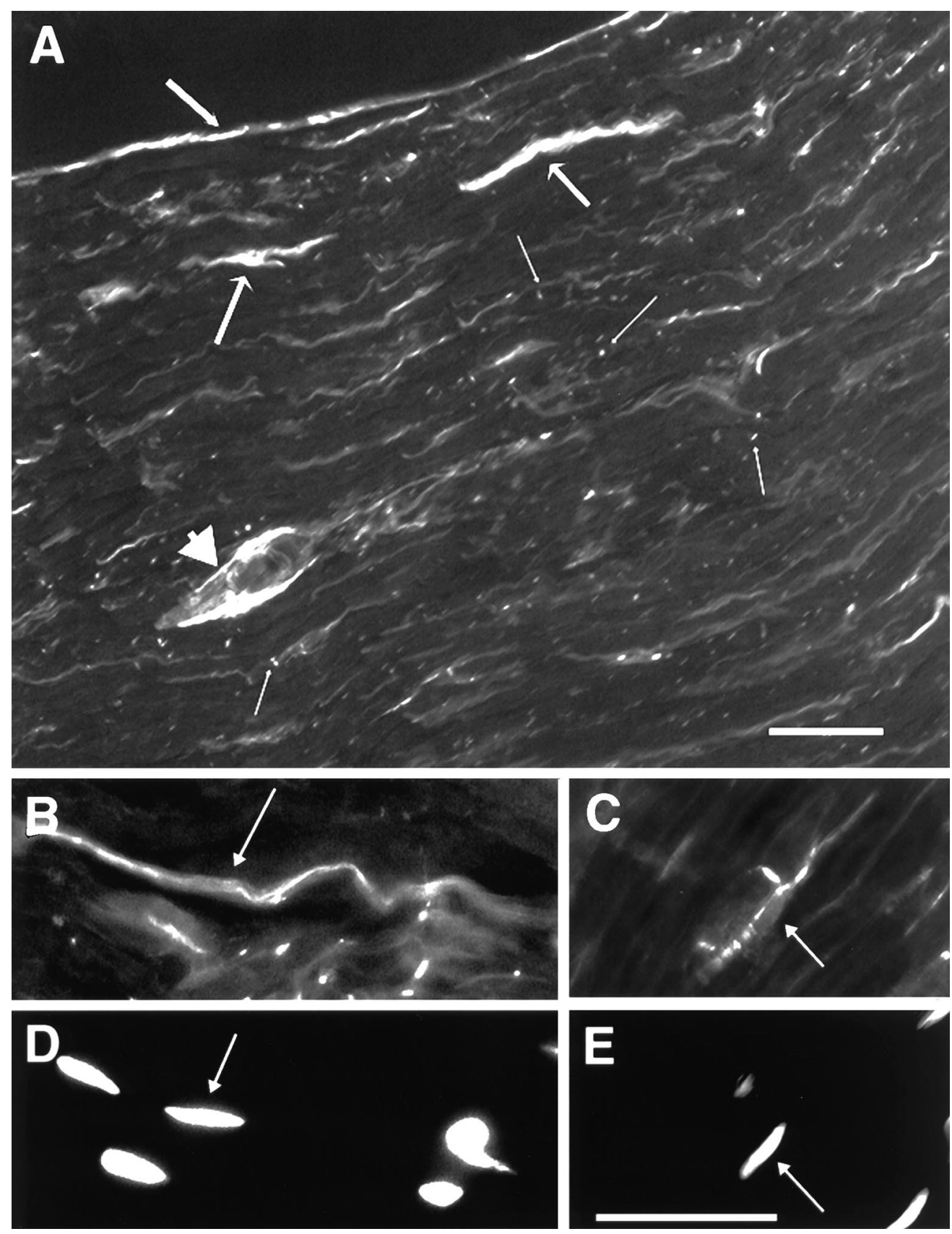

Figure 1. Localization of NG2 in adult sciatic nerve. NG2 was localized in cryostat sections of adult sciatic nerve prepared as described in Materials and Methods. A, Low-power overview of NG2 immunoreactivity in adult nerve. NG2 is associated with the extracellular matrix surrounding the nerve (smallheaded arrow), linear cellular elements within the nerve (wide-headed arrow), large blood vessels (large arrowhead), and numerous small puncta (small thin arrows). Scale bar, $100 \mu \mathrm{m} . B, C$, Highpower views of NG2-positive cells from adult sciatic nerve. These cells, which are stained at their plasma membrane with the anti-NG2 antibodies, have elongated, thin processes that lie between the nerve fibers. $D, E$, Hoechst 33258-stained nuclei of the cells shown in $B$ and $C$. Scale bar $(E), 50 \mu \mathrm{m}$. glycoprotein (MAG) (Fig. 2A-C) and myelin basic protein (MBP) (Fig. 2D-F), two markers for myelinating Schwann cells. When the images of the two different fluorochromes were colorized and digitally merged, the NG2-positive profiles (Fig. 2, arrows) appeared to fill the space between the myelinating Schwann cells. Although the myelin sheath did not bind the anti-NG2 antibodies, a thin rim of light staining was observed along the external surface of the Schwann cell. This rim of anti-NG2 immunoreactivity is associated with either the Schwann cell membrane or the basal lamina that surrounds the Schwann cell-axon unit. We also used a monoclonal antibody against the S100 $\beta$ protein as a general marker for Schwann cells (Jessen and Mirsky, 1991). As shown in Figure 2, $E-G$, there was again little or no overlap between the distribution of NG2 and that of $\mathrm{S} 100 \beta$ in adult sciatic nerve. Last, we compared the distribution of NG2 with that of the low-affinity neurotrophin receptor p75 (Fig.
$2 J-L)$. The anti-p75 antibody only lightly stained the Schwann cell cytoplasm and myelin but was enriched in the Schwann cell microvilli that abut the node of Ranvier (also see Fig. 5). None of these structures expressed high levels of NG2. Nevertheless, there was a low level of anti-p75 staining associated with the NG2positive cells. This low level of double staining was not unexpected, because perineurial fibroblasts and numerous other cell types are reported to express p75 (Bothwell, 1991; Bradley et al., 1998). Because neither Schwann cells nor the myelin they make contains the NG2 proteoglycan, these data suggest that the major source of NG2 in adult peripheral nerve is the perineurial fibroblasts. Because these single- and double-label studies only used adult nerve, we cannot rule out the possibility that other cell types produce NG2 at earlier developmental stages (Schneider et al., 2001).

Because cellular identification is often difficult in intact tissue, 

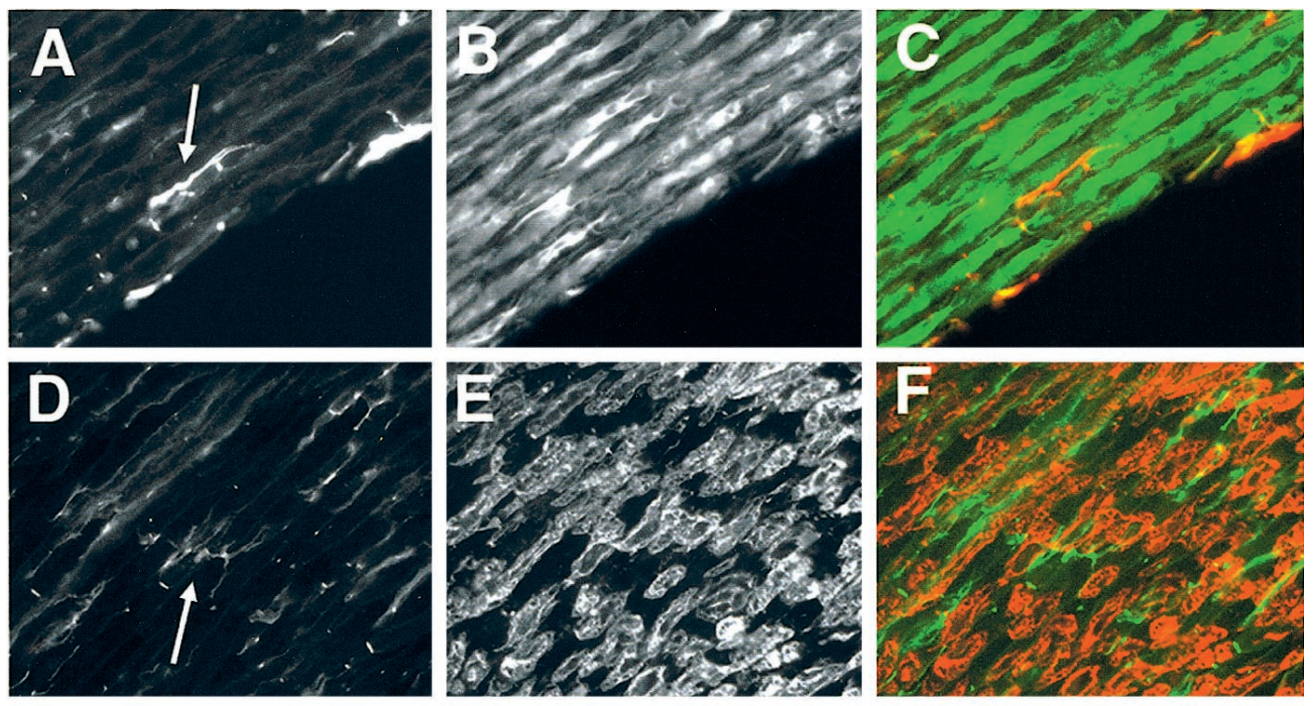

Figure 2. Comparison of the localization of NG2, MBP, MAG, S100 $\beta$, and p75 in adult sciatic nerve. Tissue sections were stained as described in $\mathrm{Ma}$ terials and Methods. Each horizontal row shows staining with the individual antibodies and a colorized and digitally merged image showing both fluorochromes. $A$, Monoclonal anti-NG2; $B$, rabbit anti-MAG; $C, A$ and $B$ colorized and merged. $D$, Rabbit antiNG2; $E$, mouse anti-MBP; $F, D$ and $E$ colorized and merged. $G$, Rabbit antiNG2; $H$, mouse anti $\mathrm{S} 100 \beta ; I, G$ and $H$ colorized and merged. $J$, Mouse antiNG2; $K$, rabbit anti-p $75 ; L, J$ and $K$ colorized and merged. Neither the Schwann cells nor the myelin they make are stained with the anti-NG2 antibodies. Scale bar, $20 \mu \mathrm{m}$.
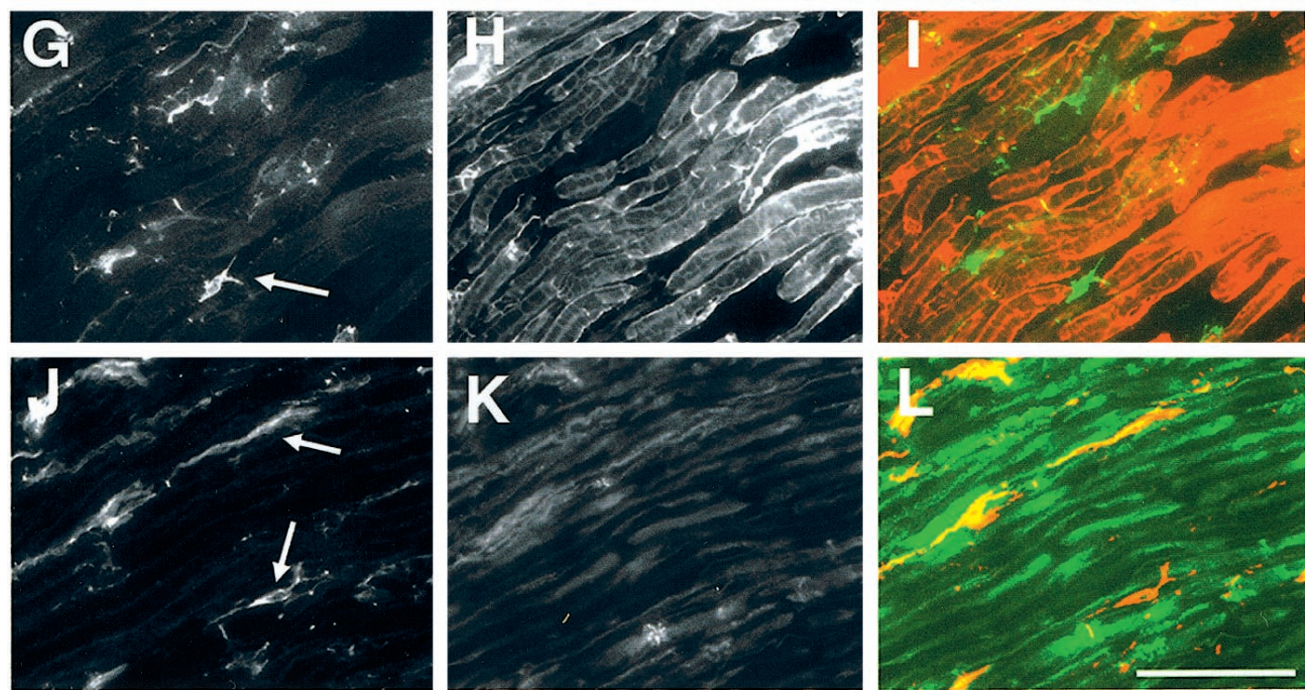

we prepared cultures of adult sciatic nerve and stained the cells with cell type-specific marker antibodies after $48 \mathrm{hr}$ in dissociated cell culture. As shown in Figure $3, A$ and $D$, the anti-NG2 antibodies bound to a population of large cells that were well spread on the substrate. In contrast, anti-p75 antibodies recognized thin cells with a spindle-shaped appearance (Fig. 3B, $G$ ). There was no overlap in the staining obtained with these two antibodies. The NG2-positive cells also bound antibodies against the thy1.1 antigen, a marker for fibroblasts and thymocytes, as well as other cell types (Brockes et al., 1979). The p75-positive Schwann cells did not bind the anti-thy1.1 antibodies. In additional experiments, the large NG2-positive cells were not stained with monoclonal antibody 04, which did stain the spindle-shaped Schwann cells (data not shown). Similar results were obtained when we stained cultures of newborn rat dorsal root ganglia that were grown for $8 \mathrm{~d}$ under conditions that foster the proliferation of non-neuronal cells. The anti-NG2 antibodies stained large, well spread cells that were not labeled with the anti-p75 antibodies (data not shown). Highly purified Schwann cells maintained in continuous culture in the presence of neuregulin and forskolin also did not bind the anti-NG2 antibodies (data not shown). Together these double-label fluorescence in vivo and in vitro studies show that NG2 is not expressed by myelinating or non- myelinating Schwann cells, but it is present on fibroblastic cells within the adult peripheral nerve.

Most cellular elements within the mature peripheral nerve are surrounded by a basal lamina (Rutka et al., 1988). Individual Schwann cell-axon units are surrounded by an endoneurial basal lamina that contains laminin B1 and B2 chains, whereas nerve bundles and the entire nerve itself are surrounded by a perineurial basal lamina that contains S-laminin (Sanes et al., 1990). Given the apparent association of NG2 with some of these basal laminae (Fig. 1), we compared the distribution of NG2 with that of the laminin B2 chain to identify those basal laminae that are enriched in NG2. As shown in Figure 4B, high levels of anti-laminin B2 immunoreactivity were found in the endoneurial basal lamina tubes surrounding the Schwann cell-axon units. These structures contain only very low levels of anti-NG2 immunoreactivity, which was often observed as punctate aggregates of immunoreactivity (Fig. 4, A,C). The endoneural basal lamina extends into the nodal gap, which was more heavily stained with the anti-NG2 antibodies (see below). The NG2-positive cellular profiles were costained with the anti-laminin B2 chain antibodies, most likely a reflection of their fibroblastic nature (Fig. 4, arrows). NG2 immunoreactivity also was found on the perineurial basal lamina (Fig. 1, smallheaded arrow), a structure that was also stained with the anti-S- 

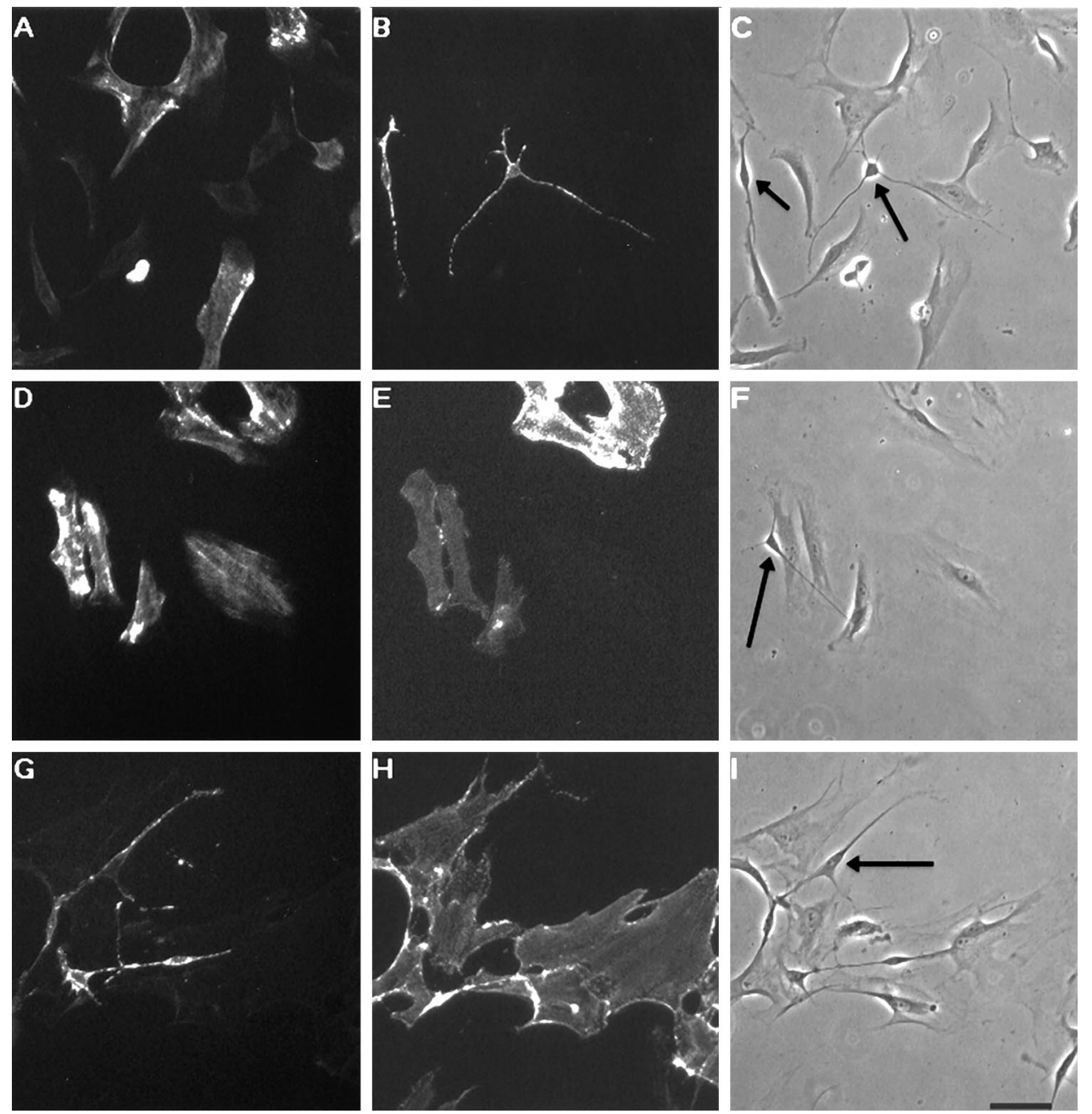

Figure 3. Lack of colocalization of NG2 and p75 in dissociated cultures of adult rat sciatic nerve. Dissociated cell cultures of rat sciatic nerve were prepared and immunofluorescently stained as described in Materials and Methods. $A$, Anti-NG2 stain; $B$, anti-p75 stain. The large, flat NG2-positive cells are p75-negative, whereas the spindleshaped cells are p75-positive. $C$, Phasecontrast view of the cultures. $D$, Anti-NG2 stain; $E$, anti-thy1.1 stain; $F$, phase contrast view. The NG2-positive cells are also stained with the anti-Thy1.1 antibodies. $G$, Anti-p75 stain; $H$, anti-thy1.1 stain; $I$, phase contrast view of the culture. The p75positive Schwann cells do not bind the antithy1.1 antibodies. The arrows in $C, F$, and $I$ point to spindle-shaped Schwann cells. Scale bar, $20 \mu \mathrm{m}$.
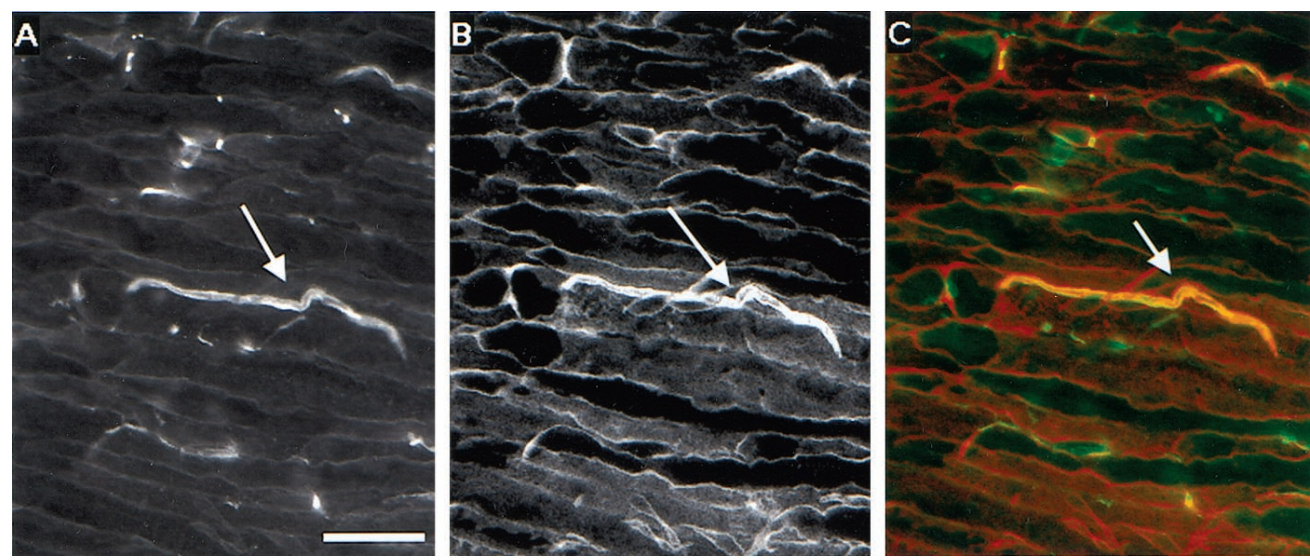

Figure 4. Comparison of the localization of NG2 with that of the endoneurial extracellular matrix. Sections of adult sciatic nerve were immunofluorescently stained as described in Materials and Methods. $A$, Anti-NG2 stain; $B$, antilaminin $\mathrm{B} 2$ stain; $C$, colorized and digitally merged image of $A$ and $B$. The endoneurial matrix is heavily stained with the anti-laminin B2 antibodies but only contains discontinuous and punctate deposits of anti-NG2 immunoreactivity. Scale bar, $10 \mu \mathrm{m}$. laminin antibodies (data not shown). The epineurial and perineurial basal laminae constitute the nerve-blood barrier, and the specific association of NG2 with these structures suggests that it may participate in these barrier functions.

The small puncta and dash-like structures within the sciatic nerve that were stained with anti-NG2 antibodies strongly resemble nodes of Ranvier. We therefore compared the distribution of NG2 with that of several different proteins known to be specifically associated with nodes of Ranvier. As shown in Figure 5, A and $B, \mathrm{NG} 2$ immunoreactivity is coincident with the staining obtained with a monoclonal antibody that recognizes the cytoplasmic domains of all known mammalian sodium channel isoforms (Rasband et al., 1999). In Figure 5, $A$ and $B$, it appears that NG2 is slightly out of alignment with the anti-sodium channel staining. Because NG2 is likely extracellular, and the epitope recognized on the sodium channel is intracellular, such misalignment would be expected if the plane of section were not exactly parallel to the longitudinal axis of the nerve. A similar close opposition and misalignment were observed (Fig. 5C,D) when sections were stained with antibodies against NG2 and ankyrin G 

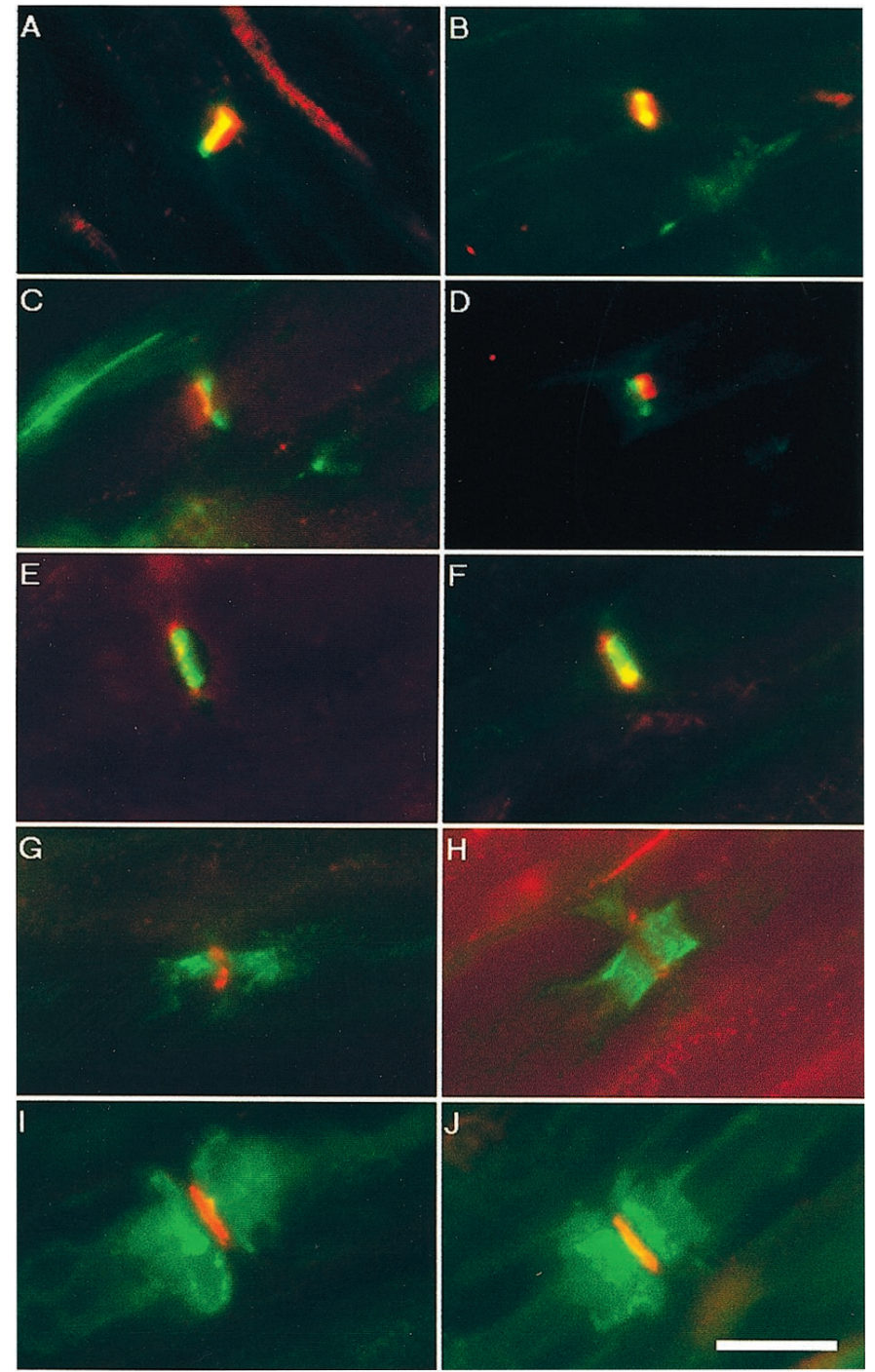

Figure 5. NG2 is found at nodes of Ranvier. Sections of adult sciatic nerve were stained with the indicated antibodies and digitally merged as described in Materials and Methods. $A, B$, Rabbit anti-NG2 (green) and mouse anti-sodium channel (red). $C, D$, Rabbit anti-NG2 (green) and mouse anti-ankyrin $\mathrm{G}$ (red). E, F, Mouse anti-NG2 (red) and rabbit anti-NrCaM (green). $G, H$, Mouse anti-NG2 (red) and rabbit anti-caspr (green). I, J, Mouse anti-NG2 (red) and rabbit anti-p75 (green). NG2 immunoreactivity colocalizes with that of the sodium channel, ankyrin $\mathrm{G}$, and $\mathrm{NrCaM}$ but is not found in the paranodal and juxtaparanodal regions that contain caspr. NG2 is also not associated with the Schwann cell microvilli that stain heavily for p75. Scale bar, $5 \mu \mathrm{m}$.

$480 / 270$, an isoform of ankyrin specifically found on the cytoplasmic surface of the nodal axolemma (Kordeli et al., 1995). NG2 immunoreactivity colocalized closely with staining for $\mathrm{NrCaM}$ (Fig. 5E,F), a member of the immunoglobulin superfamily of cell adhesion molecules that is specifically found at nodes of Ranvier in the PNS (Davis et al., 1996). In contrast to the colocalization of NG2 with these three nodal proteins, NG2 was not coincident with caspr, a marker for the paranodal region (Einheber et al., 1997). Rather, NG2 immunoreactivity appeared to fill the gap between the two closely opposed bands of caspr staining (Fig. $5 G, H)$. This filling of the nodal gap by NG2 was more apparent when sections were stained with the anti-p75 antibodies, which heavily stain the Schwann cell microvilli (Fig. 5I,J). The anti-NG2 staining of the node of Ranvier persisted in teased sciatic nerve preparations (Fig. 6), demonstrating that NG2 is bound to the node, either directly or indirectly, and that the staining pattern observed in intact nerve preparations is not simply a consequence of the three-dimensional structure of the nerve. Figure 6 also shows again that the p75-positive Schwann cells do not bind the anti-NG2 antibodies (Fig. 6E,F, arrows). Thus, NG2 is present at the node of Ranvier, where it colocalizes with other known components of the node.

The primary structure of NG2 predicts a transmembrane glycoprotein with covalently attached glycosaminoglycan (GAG) chains (Nishiyama et al., 1991). Previous studies of NG2 in the CNS have indicated that most of the NG2 present is localized to membranes (Levine and Card, 1987; Butt et al., 1999; Ong and Levine, 1999). In contrast, the data above show that NG2 can be a component of extracellular structures such as the epineurial and perineurial basal lamina. This extracellular compartmentalization is consistent with the ability of some transfected cell lines to secrete or shed a truncated form of NG2 into the medium and with the ability of NG2 to bind to extracellular matrix molecules (Nishiyama et al., 1995; Burg et al., 1996). To assess whether some of the NG2 in peripheral nerve is in a nonmembranous subcellular compartment, we divided homogenates of sciatic nerve and brainstem white matter into total soluble and particulate fractions and then assayed these fractions for NG2 using immunoblotting. Figure $7 A$, top panel, shows that considerable amounts of NG2 from sciatic nerve can be extracted into aqueous buffers, whereas lesser amounts of NG2 from CNS white mater partition into the soluble fraction. When gels such as those shown were analyzed by densitometry, the ratio of soluble to particulate NG2 in sciatic nerve was $0.91(n=3)$; in brainstem white matter, the ratio was $0.26(n=2)$. In these immunoblots, NG2 appears as single polypeptide with a molecular weight of 240,000; a high molecular weight smear that is more typical of the electrophoretic mobility of proteoglycans was not detected in extracts of sciatic nerve, although such a smear could be seen in extracts of brainstem white matter after overexposure of the x-ray film (data not shown). Although this suggests that NG2 may exist in a nonproteoglycan form, it is possible that intact proteoglycans either transfer poorly to nitrocellulose membranes or are not well detected with our antibodies. Therefore, to determine whether peripheral nerve NG2 contains chondroitin sulfate GAG chains, we prepared total nonionic detergent extracts of sciatic nerve and brainstem white matter and treated the extracts with proteasefree chondroitinase $\mathrm{ABC}$ before electrophoresis and immunoblotting. This procedure removes most of the GAG chains, causing increased intensity of the core protein band on immunoblots (Stallcup et al., 1983). Figure 7A, bottom panel, shows that when a total extract of sciatic nerve was treated with chondroitinase $\mathrm{ABC}$, there was a $165 \%$ increase in the intensity of the core protein bands. Similar treatment of a nonionic detergent extract of brainstem white matter, however, resulted in a $335 \%$ increase in the intensity of the core protein band. These data suggest that in peripheral nerve, approximately half of the NG2 present is in a soluble as opposed to a membrane-bound compartment, and NG2 is not as heavily decorated with chondroitin sulfate GAG chains as are the forms of NG2 found in the CNS.

The data given above suggest that in peripheral nerve, perineurial fibroblasts synthesize and secrete NG2, which then becomes localized to nodes of Ranvier and other structures. This model predicts that some of NG2 might be in a subcellular compartment that also contains other proteins associated with the nodal region. To isolate such a subcellular compartment, we 

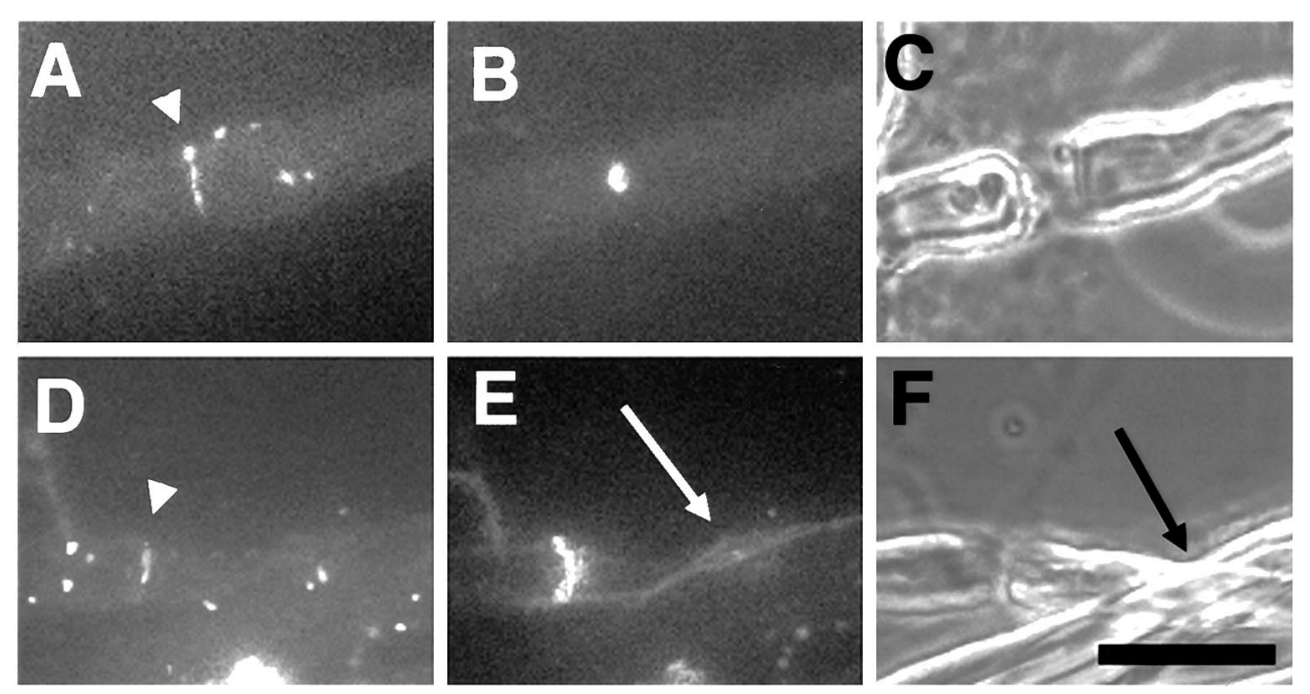

Figure 6. NG2 is localized at nodes of Ranvier in teased sciatic nerve fibers. Teased sciatic nerve fibers were stained with the indicated antibodies. $A$, Rabbit anti-NG2; $B$, mouse anti-sodium channel; $C$, phase contrast; $D$, mouse anti-NG2; $E$, rabbit anti-p75; $F$, phase contrast. NG2 (arrowhead) is concentrated at nodes of Ranvier, which also stain with the antisodium channel and anti-p75 antibodies. The arrows in $E$ and $F$ point to a p75positive spindle-shaped cell that is NG2negative. Scale bar, $10 \mu \mathrm{m}$. subjected isotonic homogenates of sciatic nerve to subcellular fractionation on sucrose gradients and obtained three membrane fractions, termed light, medium, and heavy membranes on the basis of their migration in discontinuous sucrose gradients. As shown in Figure $7 B, \mathrm{NG} 2$ is enriched in the medium $(M)$ and heavy $(H)$ membrane fractions and is not present in either peripheral nerve myelin $(M y)$ or the light membrane fraction $(L)$. Trace amounts of NG2 were detected in the material that sediments through $35 \%$ sucrose $(P)$. These medium and heavy membrane fractions also contained $\mathrm{NrCaM}$ and caspr, although smaller amounts of these proteins are also found in the other fractions analyzed (Fig. 7). In sciatic nerve, $\mathrm{NrCam}$ is present as two polypeptides with molecular weights of 135,000 and 100,000. These variant forms may arise from alternate mRNA splicing (Wang et al., 1998). Voltage-dependent sodium channels, on the other hand, were found almost exclusively in the medium membrane fraction. Although this medium-density membrane fraction represented only between 6 and $8 \%$ of the total membrane protein in sciatic nerve homogenates, it contains significant amounts of nodal membranes, together with the axolemma of unmyelinated axons. These data also establish that the NG2 proteoglycan remains associated with nodal structures after tissue disruption.

\section{DISCUSSION}

Schwann cells, perineurial fibroblasts, and blood vessels constitute the non-neuronal cellular elements of a peripheral nerve. The Schwann cells and perineurial fibroblasts cooperate to elaborate the extracellular matrices and basal laminae that surround the entire nerve, individual nerve fascicles, and each Schwann cell-axon unit (Bunge, 1993; Obremski and Bunge, 1993). The epineurial and perineurial linings form barriers whose integrity is essential in keeping pathogens out of the nerve (for review, see Olsson, 1990). The data presented here add NG2, a structurally unique, highly conserved, integral membrane protein that is most often found as a CSPG, to the growing list of cell surface and extracellular molecules found in peripheral nerve (Nishiyama et al., 1991; Arroyo and Scherer, 2000; Peles and Salzer, 2000). NG2 is likely synthesized by the perineurial fibroblasts and, when secreted or shed from the cell surface, associates with the node of Ranvier and the epineurial and perineurial basal lamina. By virtue of its anti-adhesive and growth-inhibitory properties (Dou and Levine, 1994; Fidler et al., 1999), NG2 may contribute to the barrier functions of these basal laminae and to the organization and stabilization of the node of Ranvier.

Schwann cells in vitro, grown acutely in dissociated cultures of adult sciatic nerve or for long periods in continuous culture, do not bind the anti-NG2 antibodies, and NG2 could not be detected by immunoblotting using either lysates of Schwann cell cultures or medium conditioned by Schwann cells (Morganstern et al., 1999). In contrast, abundant NG2 was detected in both perineurial fibroblast cultures and in the medium conditioned by these cells. In tissue sections, a low level of anti-NG2 immunoreactivity was associated with the outer membrane of myelinating Schwann cells. Whether this represents a low level of synthesis of NG2 by Schwann cells or the binding of secreted NG2 to sites on either the Schwann cell surface or the endoneurial basal lamina remains to be determined.

Our conclusion that the major site of NG2 expression in adult peripheral nerve is the perineurial fibroblast differs from that of Schneider et al. (2001), who suggested that in the adult mouse, AN2, the mouse homolog of NG2, is associated with a subpopulation of nonmyelinating Schwann cells. In the adult mouse, AN2 is found on blood vessels, perineurial cells, and long, thin cells that lie between myelinated axons, a distribution remarkably similar to that reported here for NG2 in the adult rat. The identification of the elongated murine cells as nonmyelinating Schwann cells is based, at least in part, on the colocalization of AN2 and p75. However, p75 can be expressed by many different cell types, including fibroblasts of peripheral nerve (Thomson et al., 1988; Bothwell, 1991; Bradley et al., 1998). In addition, after nerve crush, when Schwann cells revert to an immature and nonmyelinating phenotype, there is no increase in levels of either AN2 or NG2 (Morganstern et al., 1999; Schneider et al., 2001). Such an increase might be expected if nonmyelinating Schwann cells expressed these two highly related antigens. Thus, although Schwann cell precursors can express NG2 and AN2 (Schneider et al., 2001), the morphological data presented here and elsewhere (Morganstern et al., 1999) suggest that perineurial fibroblasts are the major but not necessarily the only source of these molecules in normal adult nerve.

Biochemical studies of transfected cells have shown that the NG2 core protein, an integral membrane protein with a molecular weight of 300,000, can be proteolytically cleaved from the cell surface to generate truncated molecules (Nishiyama et al., 1995). 
A

\section{sciatic brainstem}
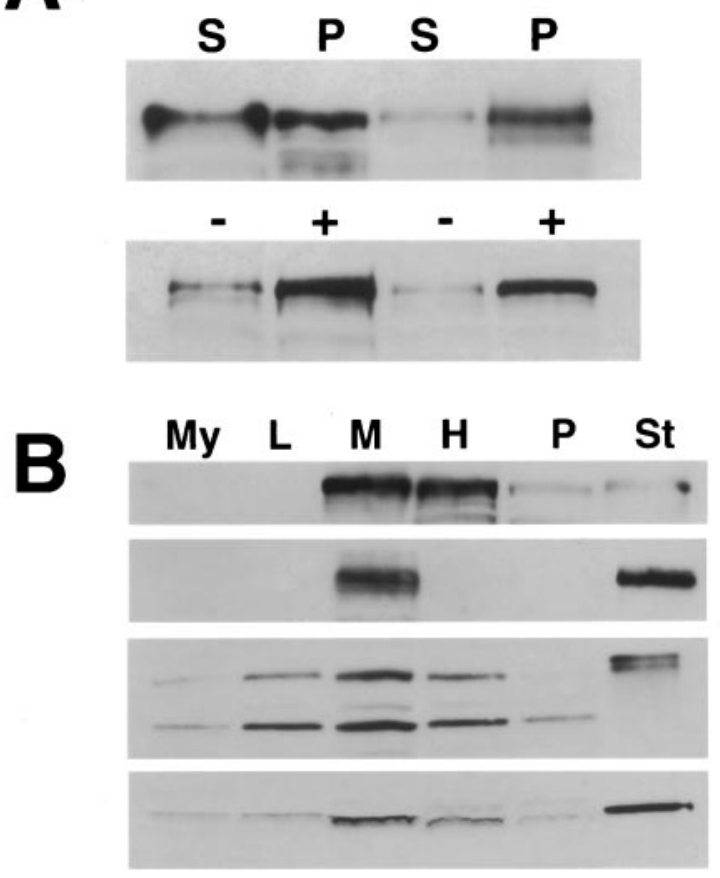

NG2

$\mathrm{Nav}$

NrCam

\section{caspr}

Figure 7. Biochemical identification of NG2 in sciatic nerve. A, Sciatic nerves and brainstem white matter were homogenized and differentially extracted as described in Materials and Methods. Fifty micrograms of total protein were separated on $6 \%$ SDS-polyacrylamide gels, transferred to nitrocellulose, and immunoblotted using rabbit anti-NG2. Top panel, NG2 is a major component present in total soluble extracts of sciatic nerve but only a minor component in soluble extracts of brainstem white matter. $S$, Soluble extract; $P$, particulate extract. Bottom panel, Effects of chondroitinase $\mathrm{ABC}$ digestion on the electrophoretic behavior of NG2 in total nonionic detergent extracts of either sciatic nerve or brainstem white matter. Chondroitinase digestion increases the apparent amount of the NG2 core protein of 300,000 . -, No treatment; +, digestion with chondroitinase ABC. $B, \mathrm{NG} 2$ cofractionates with sodium channels. Sciatic nerves were subjected to subcellular fractionation as described in Materials and Methods, and the proteins present in each fraction were identified by Western blotting. The relevant regions of each Western blot are shown, aligned with the other gels. NG2 is detected as a broad band with a molecular weight of 240,000 . Voltage-dependent sodium channels also appear heterogeneous, with a molecular weight of 215,000. Two isoforms of $\mathrm{NrCam}$ are detected in sciatic nerve, with molecular weights of 135,000 and 100,000. Caspr has an apparent molecular weight of 192,000. For NG2 and $\mathrm{NrCaM}, 20 \mu \mathrm{g}$ of total protein was loaded per lane; for caspr, $40 \mu \mathrm{g}$ of protein was loaded; and for the sodium channel, $35 \mu \mathrm{g}$ of total protein was loaded. $M y$, Myelin membranes; $L$, light membranes, i.e., those membranes collected at the $12 / 22 \%$ sucrose interface; $M$, medium-density membranes, the $22 / 27 \%$ sucrose interface; $H$, heavy membranes collected at the $27 / 35 \%$ sucrose interface; $P$, material sedimenting through $35 \%$ sucrose; $S t$, protein standards used as positive controls for the Western blots. For NG2, the standard was a total nonionic detergent extract of sciatic nerve; all other $S t$ lanes were adult rat brain membranes $(5-10 \mu \mathrm{g}$ total protein).

Our data show that in the sciatic nerve a significant fraction of NG2 can be extracted into aqueous buffers and that only $\sim 50-$ $60 \%$ of the total NG2 present is tightly bound to or associated with membranes. This soluble form of NG2 may be generated by proteolytic cleavage of intact, full-length NG2. Once secreted or shed, soluble NG2 then associates with distinct structures within the nerve, including basal laminae and nodes of Ranvier.

At present, we can only speculate about how these associations occur. NG2 binds to several different extracellular matrix mole- cules, and these molecules could be NG2 binding sites in peripheral nerve (Burg et al., 1996; Tillet et al., 1997). Binding to type $\mathrm{V}$ and VI collagen is mediated by the central extended domain of the core protein (domain 2), which is present in both membranebound and secreted NG2 (Nishiyama et al., 1995; Tillet et al., 1997). However, the disposition of NG2 in sciatic nerve is not directly correlated with the localization of these collagens. Type $\mathrm{V}$ collagens are synthesized by neonatal Schwann cells, and these chains are most prominent in young rather than adult rats such as those studied here (Chernousov et al., 2000). In human nerve, type VI collagen is associated with the epineurium, perineurium, and endoneurium (Peltonen et al., 1990). Because NG2 is associated with the perineurial and epineurial linings but is not a major component of the endoneurium, potential interactions between extracellular NG2 and type VI collagen cannot fully account for the specific anatomical localization of NG2 in the nerve. Moreover, proteolytically processed NG2 interacts with type VI collagen poorly, if at all (Nishiyama et al., 1995). Cell adhesion molecules such as neural cell adhesion molecule, neuron-glia cell adhesion molecule, and $\mathrm{NrCam}$, which are concentrated at nodes of Ranvier, as well as tenascin C, are all potential binding partners for NG2 (Rieger et al., 1986; Martini et al., 1990; Davis et al., 1996; Burg et al., 1996).

The N-terminal domain 1 of NG2 contains two laminin G domains, a protein motif found on a variety of different proteins, including caspr and agrin (Bellen et al., 1998; Missler and Südhof, 1998). These three proteins (NG2, agrin, and caspr) are found at or close to nodes of Ranvier (Reist et al., 1987; Einheber et al., 1997; Menegoz et al., 1997). Laminin G domains participate in cell-cell and cell-matrix interactions by binding directly to proteins or carbohydrates (Talts et al., 1999). Thus, it is possible that the laminin G domains of NG2 provide a site for interactions with other molecular components of the node.

The localization of NG2 at nodes of Ranvier in the PNS is particularly intriguing, because oligodendrocyte precursor cells, the major NG2-expressing cell type in the CNS, also contact nodes of Ranvier (Butt et al., 1999). Oligodendrocyte precursor cell processes are also closely associated with synapses in the rat hippocampus (Ong and Levine, 1999; Bergles et al., 2000). Because NG2 is present at both central and peripheral nodes, as well as at other sites of ion movement across membranes, it is likely to be performing one or more important functions there.

Like many other proteoglycans, NG2 is multifunctional. Among its properties, NG2 is anti-adhesive to developing neurons, inhibits axonal growth when substrate-bound, and rapidly induces the collapse of newborn dorsal root ganglia neuronal growth cones in vitro (Dou and Levine, 1994; Fidler et al., 1999; Ughrin et al., 1999). These properties suggest several possible functions for NG2 at the node. The axonal membrane at nodes of Ranvier is capable of rapidly forming a new growth cone, and new sprouts often form here after nerve injury (Friede and Bischhausen, 1980; McQuarrie, 1985). The inhibitory properties of NG2 suggest that one function in nerve may be to prevent unregulated sprouting in normal, undamaged animals. When the nerve is injured, regenerating axons grow in a narrow space between the Schwann cell surface and the endoneurial basal lamina (the bands of Büngner; Nathaniel and Pease, 1963; Ide et al., 1983; Fawcett and Keynes, 1990). These structures contain relatively little NG2 but are enriched in growth-promoting molecules such as laminin (Ide et al., 1983). A second possible function for NG2 on the surface of perineurial cells would be to help direct filopodia into these growth-permissive conduits. 
The anti-adhesive properties of NG2 suggest a third function for NG2 at nodes of Ranvier. Our current understanding of the development of the node of Ranvier in the PNS emphasizes the essential role of the Schwann cell as a determinant of where high-density clusters of sodium channels form (Vabnick and Shrager, 1998; Peles and Salzer, 2000). In developing nerves, Schwann cells adhere to axons and elongate as they begin to express myelin-specific proteins such as MAG (Lambert et al., 1997). During this elongation phase, broad clusters of sodium channels are seen at the lateral edges of the Schwann cell. The continued elongation of the Schwann cell results in the formation of compact, high-density clusters of sodium channels, again at the lateral edges of the Schwann cell. These clusters eventually fuse to form nodal clusters. This model raises the question of what causes the elongation of Schwann cells to cease. Secreted NG2 is ideally suited to generate such a stop signal to the Schwann cell. The central extended domain of NG2 may bind to some as yet unidentified binding protein at the node exposing the globular N-terminal domain and the juxtamembrane domain (Tillet et al., 1997). Because both of these domains (domains 1 and 3) are each sufficient to inhibit axonal extension and to induce growth cone collapse (Ughrin et al., 1999), it is possible that they limit or stop the lateral extension of the Schwann cell. In this way, the deposition of NG2 at nodes of Ranvier, which occurs at approximately postnatal day 10 in the rat (J. M. Levine and A. K. Levine, unpublished observation), could function to stabilize the structure of the node.

\section{REFERENCES}

Arroyo EJ, Scherer SS (2000) On the molecular architecture of myelinated fibers. Histochem Cell Biol 113:1-18.

Bellen HJ, Lu Y, Beckstead R, Bhat MA (1998) Neurexin IV, caspr and paranodin-novel members of the neurexin family: encounters of axons and glia. Trends Neurosci 21:444-449.

Bergles DE, Roberts JD, Somogyi P, Jahr CE (2000) Glutamatergic synapses on oligodendrocyte precursor cells in the hippocampus. Nature 405:187-191.

Bothwell M (1991) Tissue localization of nerve growth factor and nerve growth factor receptors. Curr Top Microbiol Immunol 165:55-70.

Bradley JL, Abernethy DA, King RHM, Muddle JR, Thomas PK (1998) Neural architecture in transected rabbit sciatic nerve after prolonged nonreinnervation. J Anat 192:529-538.

Brockes J, Fields K, Raff MC (1979) Studies on cultured rat Schwann cells. I. Establishment of purified populations from cultures of peripheral nerve. Brain Res 165:105-118.

Bunge MB (1993) Schwann cell regulation of extracellular matrix synthesis and assembly. In: Peripheral neuropathy (Dyck PJ, Thomas PK, Low PA, Poduslo JF, eds), Ed 3, Vol 1, pp 299-316. Philadelphia: Saunders.

Burg MA, Tillet E, Timpl R, Stallcup WB (1996) Binding of the NG2 proteoglycan to type VI collagen and other extracellular matrix molecules. J Biol Chem 271:26110-26116.

Butt AM, Duncan A, Hornby MF, Kirvell SL, Hunter A, Levine JM, Berry M (1999) Cells expressing the NG2 antigen contact nodes of Ranvier in adult CNS white matter. Glia 26:92-96.

Caldwell JH, Schaller KL, Lasher RS, Peles E, Levinson SR (2000) Sodium channel $\mathrm{Na}_{\mathrm{v}} 1.6$ is loclaized at nodes of Ranvier, dendrites and synapses. Proc Natl Acad Sci USA 97:5616-5620.

Chernousov MA, Rothblum, K, Tyler WA, Stahl RC, Carey DJ (2000) Schwann cell synthesize type V collagen that contains a novel $\alpha 4$ chain. J Biol Chem 275:28208-28215.

Davis JQ, Lambert S, Bennett V (1996) Molecular composition of the node of Ranvier: identification of ankyrin-binding cell adhesion molecules neurofascin (mucin +/third FNIII domain) and NrCAM at nodal axon segments. J Cell Biol 135:1355-1367.

Dou C, Levine JM (1994) Inhibition of neurite growth by the NG2 chondroitin-sulfate proteoglycan. J Neurosci 14:7616-7628.

Einheber S, Zanazzi G, Ching W, Scherer S, Milner TA, Peles E, Salzer JL (1997) The axonal membrane protein Caspr, a homologue of neurexin IV, is a component of the septate-like paranodal junctions that assemble during myelination. J Cell Biol 139:1495-1506.

Fawcett JW, Keynes RJ (1990) Peripheral nerve regeneration. Annu Rev Neurosci 13:43-60.

Fidler PS, Schuette, K, Powell EM, Asher RA, Muir E, Levine JM,
Geller HM, Rogers JH, Faissner A, Fawcett JW (1999) Comparing astrocytic cell lines that are inhibitory or permissive for axon growth: the major axon-inhibitory proteoglycan is NG2. J Neurosci 19:8778-8788.

Fitch MT, Silver J (1997) Glial cell extracellular matrix: boundaries for axon growth in development and regeneration. Cell Tissue Res 290:379-384.

Friede RL, Bischhausen R (1980) The fine structure of stumps of transected nerve fibers in subserial sections. J Neurol Sci 44:181-203.

Ide C, Tohyama K, Nitatori T, Onodera S (1983) Schwann cell basal lamina and nerve regeneration. Brain Res 288:61-75.

Jessen KR, Mirsky R (1991) Schwann cell precursors and their development. Glia 4:185-194.

Kordeli E, Lambert S, Bennett V (1995) Ankyrin G. A new ankyrin gene with neural-specific isoforms localized at the axonal initial segment and node of Ranvier. J Cell Biol 270:2352-2359.

Lambert S, Davis JQ, Bennett V (1997) Morphogenesis of the node of Ranvier, coclusters of ankyrin and ankyrin binding proteins define early developmental intermediates. J Neurosci 15:7025-7036.

Levine J, Card JP (1987) Light and electron microscope localization a cell surface antigen (NG2) in the rat cerebellum: association with smooth protoplasmic astrocytes. J Neurosci 7:2711-2720.

Levine JM, Nishiyama A (1996) The NG2 chondroitin sulfate proteoglycan; a multifunctional proteoglycan associated with immature cells. Perspect Dev Neurobiol 3:245-259.

Levine JM, Stincone F, Lee YS (1993) Development and differentiation of glial precursor cells in the rat cerebellum. Glia 7:307-321.

Levine JM, Enquist LW, Card JP (1998) Reactions of oligodendrocyte precursor cells to alpha herpesvirus infection of the central nervous system. Glia 23:316-328.

Malhotra JD, Kazan-Gillespie K, Hortsch M, Isom LL (2000) Sodium channel $\beta$ subunits mediate homophilic cell adhesion and recruit ankyrin to points of cell-cell contact. J Biol Chem 275:11383-11388.

Martini R, Schachner M, Faissner A (1990) Enhanced expression of the extracellular matrix molecule $\mathrm{J} 1 /$ tenascin in the regenerating adult mouse sciatic nerve. J Neurocytol 19:601-616.

McQuarrie IG (1985) Effect of a conditioning lesion on axonal sprout formation at nodes of Ranvier. J Comp Neurol 231:239-249.

Menegoz M, Gaspar P, Le Bert M, Galvez T, Burgaya F, Palfrey C, Ezan P, Arnos F, Girault JA (1997) Paranodin, a glycoprotein of neuronal paranodal membranes. Neuron 19:319-331.

Mi H, Deerinck TJ, Ellisman MH, Schwarz TL (1995) Differential distribution of closely related potassium channels in rat Schwann cells J Neurosci 15:3761-3774.

Missler M, Südhof TC (1998) Neurexins: three genes and 1001 products. Trends Genet 14:20-26.

Morganstern DA, Asher RA, Levine JM, Fawcet JW (1999) Expression of the chondroitin sulfate proteoglycan NG2 in normal and regenerating peripheral nerve. Soc Neurosci Abstr 25:1264.

Nathaniel EJ, Pease DC (1963) Regenerative changes in rat dorsal roots following Wallerian degeneration. J Ultrastruct Res 9:533-549.

Nishiyama A, Dahlin KJ, Prince JT, Johnstone SR, Stallcup WB (1991) The primary structure of NG2: a novel membrane spanning proteoglycan. J Cell Biol 114:359-371.

Nishiyama A, Lin XH, Stallcup WB (1995) Generation of truncated forms of the NG2 proteoglycan by cell surface proteolysis. Mol Biol Cell 6:1819-1832.

Obremski VJ, Bunge MB (1995) Addition of purified basal lamina molecules enables Schwann cell ensheathment of sympathetic neurites in culture. Dev Biol 168:124-137.

Olsson Y (1990) Microenvironment of the peripheral nervous system under normal and pathological conditions. Crit Rev Neurosci 3:265-311.

Ong WY, Levine JM (1999) A light and electron microscopic analysis of NG2 positive oligodendrocyte precursor cells in the normal and kainate lesioned hippocampus. Neuroscience 92:83-95.

Peles E, Salzer JL (2000) Molecular domains of myelinated axons. Curr Opin Neurobiol 10:558-565.

Peltonen J, Jaakkola S, Virtanen I, Pelliniemi L (1987) Perineurial cells in culture, an immunocytochemical and electron microscopic study. Lab Invest 57:480-488.

Peltonen J, Jaakkola S, Hsiao LL, Timpl R, Chu ML, Uitto J (1990) Type VI collagen, in situ hybridization and imnohistochemistry reveal abundant mRNA and protein levels in human neurofibroma, schwannoma and normal peripheral nerve tissues. Lab Invest 62:487-492.

Rasband MN, Shrager P (2000) Ion channel sequestration in central nervous system axons. J Physiol (Lond) 525:63-73.

Rasband MN, Trimmer JS, Schwarz TL, Levinson SR, Ellisman MH, Schachner M, Shrager P (1998) Potassium channel distribution, clustering, and function in remyelinating rat axons. J Neurosci 18:36-47.

Rasband MN, Peles E, Trimmer JS, Levinson SR, Lux SE, Shrager P (1999) Dependence of nodal sodium channel clustering on paranodal axoglial contact in the developing CNS. J Neurosci 19:7516-7528.

Reist N, Magill C, McMahon UJ (1987) Agrin-like molecules at synaptic 
sites in normal, denervated and damaged skeletal muscles. J Cell Biol 105:2457-2469.

Rieger F, Daniloff JK, Pincon-Raymond M, Crossin KL, Grumet M, Edelman GM (1986) Neuronal cell adhesion molecules and cytotactin are colocalized at nodes of Ranvier. J Cell Biol 103:379-391.

Rios J, Melendez-Vasquez CV, Einheber S, Lustig M, Grumet M, Hemperley J, Peles E, Salzer JL (2000) Caspr and contactin form a complex that is targeted to the paranodal junctions during myelination. J Neurosci 20:8354-8364.

Rosenbluth J (1976) Intramembranous particle distribution at the node of Ranvier and adjacent axolemma in myelinated axons of the frog brain. J Neurocytol 5:731-745.

Rutka JT, Apodaca G, Stern R, Rosenblum M (1988) The extracellular matrix of the central and peripheral nervous system: structure and function. J Neurosurg 69:155-170.

Sanes JR, Engvall E, Butkowski R, Hunter DD (1990) Molecular heterogeneity of basal laminae: isoforms of laminin and collagenIV at the neuromuscular junction and elsewhere. J Cell Biol 111:1685-1699.

Schneider S, Bosse F, D'Urso D, Muller H-W, Sereda MW, Nave K-A, Neihaus A, Kempf T, Schnolxer M, Trotter J (2001) The AN2 protein is a marker for the Schwann cell lineage expressed by immature and nonmyelinating Schwann cells. J Neurosci 21:920-933.

Stallcup WB, Beasley LL, Levine J (1983) Cell surface molecules that characterize different stages in the development of cerebellar interneurons. Cold Spring Harb Symp Quant Biol 48:761-774.

Talts JF, Andac Z, Gohring W, Brancaccio A, Timpl R (1999) Binding of the $\mathrm{G}$ domains of laminin $\alpha 1$ and $\alpha 2$ chains and perlecan to heparin, sulfatides, $\alpha$-dystroglycan and several extracellular matrix proteins. EMBO J 18:863-870.

Thomson TM, Rettig WJ, Chesa PG, Green SH, Mena AC, Olds LJ (1988) Expression of human nerve growth factor receptor on cells derived from all three germ layers. Exp Cell Res 174:533-539.

Tillet E, Ruggiero F, Nishiyama A, Stallcup WB (1997) The membranespanning proteoglycan NG2 binds to collagens V and VI through the central nonglobular domain of its core protein. J Biol Chem 272:10769-10776.

Ughrin Y, Guo W, Levine JM (1999) Multiple globular domains of the NG2 proteoglycan core protein mediate axon growth inhibition. Soc Neurosci Abstr 25:748.

Vabnick I, Shrager P (1998) Ion channel redistribution and function during development of the myelinated axon. J Neurobiol 37:80-96.

Wang B, Williams H, Du JS, Terrett J, Kendwrick S (1998) Alternate splicing of human NrCAM in neural and nonneural tissues. Mol Cell Neurosci 10:287-295.

Wang H, Kunkel DD, Martin TM, Schwartzkroin PA, Tempel BL (1993) Heteromultimeric $\mathrm{K}+$ channels in terminal and juxtaparanodal regions of neurons. Nature 365:75-79. 